\title{
Analyse de la Diversité Floristique de Quelques îles Aménagées du Barrage de Buyo (Côte d'Ivoire)
}

\author{
Soro Yenilougo,
}

UFR des Sciences de la Nature (SN), Université Nangui Abrogoua, Abidjan, Côte d'Ivoire

\section{Ouattara Noufou Doudjo,}

UFR des Sciences de la Nature (SN), Université Nangui Abrogoua, Abidjan, Côte d'Ivoire, Centre Suisse de Recherches Scientifiques, Côte d'Ivoire

\section{Missa Koffi,}

Centre de Recherche en Ecologie, Abidjan, Université Nangui Abrogoua,

Côte d'Ivoire, Centre Suisse de Recherches Scientifiques, Côte d'Ivoire

\section{Bakayoko Adama,}

UFR des Sciences de la Nature (SN), Université Nangui Abrogoua, Abidjan, Côte d'Ivoire, Centre Suisse de Recherches Scientifiques, Côte d'Ivoire

Doi: 10.19044/esj.2019.v15n18p165 URL:http://dx.doi.org/10.19044/esj.2019.v15n18p165

\section{Résumé}

La présente étude se propose d'analyser la composition et la diversité floristique des îles du barrage de Buyo après forte anthropisation. À l'aide de relevé de surface, de relevé linéaire et de relevé itinérant, la composition et la diversité floristique de onze îles ont été déterminées. L'inventaire a permis de noter 723 espèces de plantes reparties entre 353 genres et 99 familles. L'importance écologique des espèces a été appréciée à partir de l'Indice de Valeur d'Importance. L'étude a montré que pour les arbres de diamètre $\geq 10$ $\mathrm{cm}$, les espèces Alchornea cordifolia, Ceiba pentandra, Theobroma cacao, Lannea welwitschii sont celles qui ont les plus forts indices de valeur d'importance des espèces. La moyenne de la richesse spécifique montre une différence significative $(p \leq 0,03797)$ entre les différentes îles du barrage de Buyo. L'île D, proche de la forêt du Parc, avec une présence moyenne de mammifères, est la plus riche en espèces $(101 \pm 10,74)$. Les îles Apollinaire et Laminebougou, plus éloignées de la forêt mère et avec une intense activité, sont les plus pauvres en espèces $(51 \pm 11,14 ; 50 \pm 6,29)$. La diversité floristique et l'équitabilité ne varient pas statistiquement entre les îles.

Mots-clés : Diversité floristique, îles du barrage de Buyo, Anthropisation, Conservation 


\title{
Analysis of Floristic Diversity of Some Adjusted Island of Buyo's Dam (Ivory Coast)
}

\author{
Soro Yenilougo, \\ UFR des Sciences de la Nature (SN), \\ Université Nangui Abrogoua, Abidjan, Côte d'Ivoire \\ Ouattara Noufou Doudjo,
}

UFR des Sciences de la Nature (SN), Université Nangui Abrogoua, Abidjan, Côte d'Ivoire, Centre Suisse de Recherches Scientifiques, Côte d'Ivoire

Missa Koffi,

Centre de Recherche en Ecologie, Abidjan, Université Nangui Abrogoua,

Côte d'Ivoire, Centre Suisse de Recherches Scientifiques, Côte d'Ivoire

\section{Bakayoko Adama,}

UFR des Sciences de la Nature (SN), Université Nangui Abrogoua, Abidjan, Côte d'Ivoire, Centre Suisse de Recherches Scientifiques, Côte d'Ivoire

\begin{abstract}
This paper focuses on analyzing the floristic composition of islands of Buyo's dam after strong anthropization. Using surfaces surveys, linear surveys and itinerant surveys, the floristic composition and diversity were determined. Seven hundred and twenty-three plants species belonging to 353 genera and 99 families were recorded. The ecological importance of the species has been assessed from the Value of Significance. The study showed that for tree species with diameter $\geq 10 \mathrm{~cm}$, the species Alchornea cordifolia, Ceiba pentandra, Theobroma cacao, and Lannea welwitschii were the richest and had the highest Indices of Value Importance. The mean richness of species showed a significant difference $(p \leq 0$. 03797) among the Islands of Buyo's dam. Island D which is close to the Park with the presence of animals is rich in species $(101 \pm 1074)$. Apolinaire and Laminebougou Islands farthest to the Park with strong agricultural activity are poor in species $(51 \pm 11.14$; $50 \pm 6$. 29). Floristic diversity and evenness do not vary statistically from one site to another.
\end{abstract}

Keywords: Floristic diversity, Islands of Buyo's dam, Anthropization, conservation 


\section{Introduction}

Situé au Sud-ouest de la Côte d'Ivoire, le Parc national de Taï occupe à lui seul plus de $50 \%$ de la superficie totale des zones forestières ouestafricaines bénéficiant d'un statut de haute protection (Koffi, 2000). On estime que $54 \%$ de sa flore est endémique (Martin, 1991), ce qui fait de ce vestige, un espace remarquable de sauvegarde de la biodiversité. Ainsi, de nombreux travaux de recherche d'inventaire botanique ont montré le caractère exceptionnel de cette forêt (Guillaumet \& Adjanohoun, 1971 ; Aké -Assi, 1984 ; Chatelain et al., 1996 ; Aké -Assi, 2001, 2002 ; Adou et al., 2005). Cependant, ce site n'échappe pas aux énormes pressions anthropiques, surtout à sa périphérie Nord-ouest, qui est soumise à une forte dégradation. Les causes les plus courantes sont l'abattage des arbres, l'agriculture itinérante, le développement de l'agriculture familiale en agriculture industrielle (FAO, 2003), l'exploitation minière, la recherche du bois de chauffe, ainsi que la création du barrage de Buyo.

Depuis sa création en 1980, le lac, occasionné par le barrage, a submergé d'importantes zones écologiques (N'Guessan, 2001), créant ainsi des îles dans la zone Nord-ouest du Parc. Cette zone, qui formait un seul bloc avant la création du barrage, est aujourd'hui constituée d'eau et d'îles formées par les terres en altitude, dont la plupart sont des collines comportant des îlots forestiers. En plus de l'inondation, ces îlots forestiers ont connu des perturbations liées à l'occupation par les paysans.

À la suite d'une reprise en main par l'Office Ivoirien des Parcs et Réserve (OIPR) des parcelles exploitées clandestinement, les paysans se sont retirés de ces îlots forestiers. Après ce retrait, les gestionnaires du Parc veulent voir la dynamique de reconstitution de ces formations forestières fortement anthropisées, d'où cette étude. Elle a pour objectif de fournir des données pouvant permettre une gestion rationnelle des îlots forestiers du barrage de Buyo, après la période d'occupation humaine.

\section{Méthodologie Site d'étude}

Les îles du barrage de Buyo sont situées dans le Nord du Parc national de Taï, localisé dans le Sud-ouest de la Côte d'Ivoire, entre les latitudes $6^{\circ} 12^{\prime}$ et $6^{\circ} 18^{\prime}$ Nord et les longitudes $7^{\circ} 06^{\prime}$ et $7^{\circ} 12^{\prime}$ Ouest (Figure 1 ). 


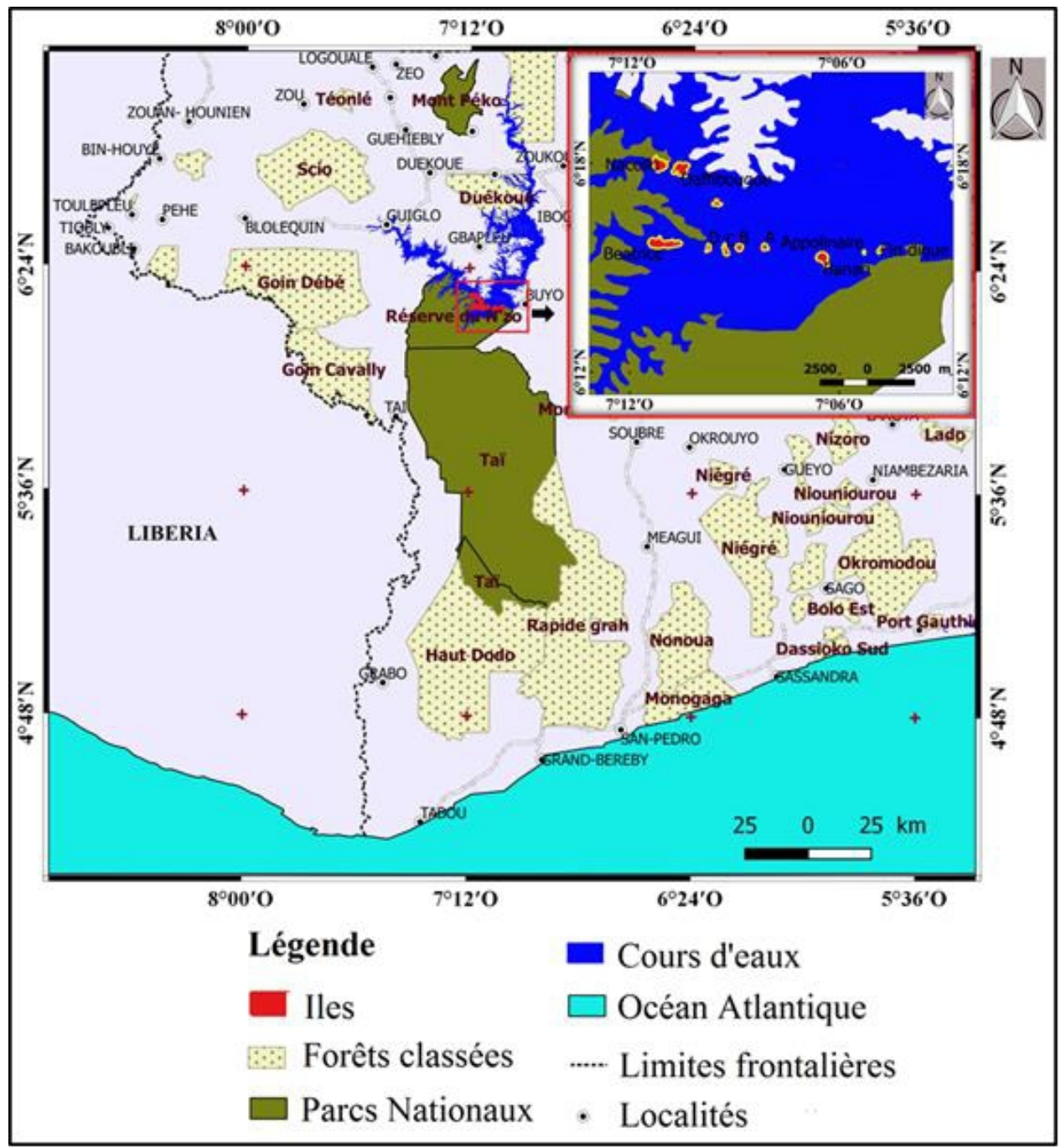

Figure 1. Localisation géographique des îles du barrage de Buyo

Le climat est, selon Van Rompaey (1993) et New et al. (2000), du type subéquatorial, avec une pluviométrie annuelle moyenne comprise entre 1750 $\mathrm{mm}$ et $1950 \mathrm{~mm}$. La durée du déficit hydrique varie de 4,5 mois au nord, à 3,5 mois au sud (Eldin, 1971). La région repose sur une roche mère principalement granitique (Bolgarsky, 1950). La végétation du Parc national de Taï fait partie des forêts denses humides sempervirentes du grand bloc éburnéo-libérien. Elle se caractérise par une flore riche, avec une forte proportion d'espèces endémiques locales qui confèrent à ces forêts un faciès particulier (Mangenot, 1955). Ces espèces, liées à une pluviométrie élevée, sont qualifiées de « sassandriennes », car elles sont limitées dans leur distribution vers le Nord-est par le fleuve Sassandra. Les formations forestières de la zone d'étude sont 
classées dans l'association à Eremospatha macrocarpa et à Diospyros mannii (Guillaumet \& Adjanohoun, 1971).

Les îles qui font 1'objet de cette étude sont au nombre de 11, et sont situées dans la réserve de faune du N'Zo, qui est la partie Nord-ouest du Parc national de Taï. Ces îles, que sont Nacollo, Daffibougou, Laminebougou, Béatrice, D, C, B, A, Banari, Apollinaire et Findique, émergent du lac du barrage de Buyo, avec des superficies inégales et des distances inégales de la forêt mère.

\section{Méthodes d'échantillonnage}

La collecte des données s'est effectuée de mars 2017 à septembre 2018. Ce qui a permis d'explorer la zone à différentes périodes de l'année, surtout au cours des crues et des décrues.

Trois méthodes de collecte de données ont été utilisées, à savoir : le relevé de surface, le relevé linéaire et le relevé itinérant.

Le relevé de surface a consisté à délimiter des surfaces carrées de $25 \mathrm{x}$ $25 \mathrm{~m}$ pour collecter des informations plus détaillées. Dans chaque placette, toutes les espèces (arbres, arbustes, lianes et herbes) ont été collectées en prenant en compte celles atteignant un diamètre d'au moins $5 \mathrm{~cm}$, à 1,30 m au-dessus du sol (Adou et al., 2005 ; N'Sielolo et al., 2015). Au total, 44 placettes ont été positionnées sur les 11 îles, en raison de 4 par île.

Quant au relevé linéaire, il a consisté à tendre horizontalement, à ras du sol, un ruban long de généralement 200 mètres (Chatelain, 1996 ; Kouamé, 1998 ; Bakayoko et al., 2004, 2011). Nous avons utilisé une longueur de 200 mètres pour les îles de grande taille, et moins de 200 mètres pour les îles de petite taille. À partir de ce dispositif, nous avons effectué des mesures à des intervalles réguliers de 2 mètres. Ainsi, la hauteur minimale et maximale de chacun des contacts avec la végétation est mesurée à l'aide d'un jalon de 8 mètres, dressé verticalement. Au-delà des 8 mètres, les valeurs de hauteur sont estimées. Dans cette étude, 11 relevés ont été effectués en raison de 1 par île.

Dans le but de compléter la liste floristique générale de chaque île, un inventaire itinérant a été effectué. Cette méthode consiste à parcourir les îles dans différentes directions et à recenser toutes les espèces végétales rencontrées, sans tenir compte de leur abondance, ni de leur taille. Pour les noms des taxons recensés, la nomenclature APG IV (2016) a été adoptée.

\section{Analyses des données}

La composition floristique d'un milieu désigne l'ensemble de toutes les espèces végétales rencontrées en ce milieu, leur abondance, leur répartition par famille, par genre, mais aussi par type biologique et type morphologique, selon Aké -Assi (1984). Les types biologiques utilisés sont ceux définis par Raunkiaer (1934) et adaptés pour l'étude des formations végétales tropicales 
par Aké -Assi (2001-2002) : les thérophytes (Th), les hémicryptophytes (Hc), les géophytes $(\mathrm{G})$, les chaméphytes $(\mathrm{Ch})$, les lianes (L) et les phanérophytes $(\mathrm{Ph})$.

La liste des espèces végétales a été confrontée à celle d'Aké -Assi (2001-2002) pour donner la répartition phytogéographique. Les principaux types chorologiques utilisés sont : Guinéo-congolaises (GC), GCW, Soudanozambéziennes (SZ), GCi, et sassandriennes.

L'Indice de Valeur d'Importance des espèces (IVI) caractérise la place qu'occupe, au sein d'une végétation, chaque espèce par rapport à l'ensemble des autres espèces. Cet indice a été mis au point par Cottam et Curtis (1956) et est fréquemment utilisé pour évaluer la prépondérance spécifique en forêts tropicales (Mori et al., 1983 ; Latour, 1994 ; Corthay, 1996 ; Kouamé, 1998 ; Bakayoko, 1999 ; Adou et al., 2005 ; Missa et al., 2015). Il est calculé selon la formule suivante :

\section{IVI= FREQesp+ DENSesp+ DOMesp}

Cet indice varie de 0 à 300 .

La fréquence relative d'une espèce (FREQesp) est le rapport de sa fréquence spécifique au total des fréquences spécifiques de toutes les espèces. La fréquence spécifique d'une espèce est le nombre de placettes dans lesquelles est présente cette espèce. La densité relative d'une espèce (DENSesp) est le rapport de sa densité absolue (c'est-à-dire le nombre d'individus par unité de surface) au total des densités absolues de toutes les espèces. La dominance relative d'une espèce (DOMesp) est le quotient de son aire basale (c'est-à-dire la surface de la section du tronc correspondant au $\mathrm{DBH})$ avec l'aire basale totale de toutes les espèces.

La diversité floristique renferme la richesse floristique et l'équitabilité, qui est la manière dont se répartissent les individus des espèces rencontrées. Pour quantifier la diversité spécifique de chaque île, nous avons calculé l'indice H' de diversité de Shannon-Weaver (Shannon \& Weaver, 1949) en $\log$ de base 2, conformément à la théorie de l'entropie, qui se mesure en bit, et l'indice d'équitabilité de Piélou EQ. L'indice de Shannon tient compte du nombre d'espèces inventoriées et de leurs abondances. Il est utilisé pour évaluer l'hétérogénéité et la diversité d'un biotope.

Dans une communauté de $\mathrm{S}$ espèces et de $\mathrm{N}$ individus, cet indice est calculé selon la formule suivante :

$\mathbf{H}^{\prime}=\sum_{\mathrm{i}=1 . . \mathrm{s}} \mathbf{N}_{\mathrm{i}} / \mathbf{N} \cdot \log _{2}\left(\mathbf{N}_{\mathrm{i}} / \mathbf{N}\right)$

$\mathbf{N}:$ le nombre total d'espèces

$\mathbf{N}_{\mathrm{i}}$ : le nombre d'individus de l'espèce $\mathrm{i}$

$E Q=H^{\prime} / \log _{2} N_{0}$

$\mathrm{N}_{0}$ : nombre total d'espèces 


\section{Analyse statistique}

L'analyse de variance à un facteur réalisé avec le logiciel $\mathrm{R}$, a permis de comparer : (i) le nombre d'espèces des îles ; (ii) la diversité des îles et (iii) l'équitabilité des îles. Le test de Tuckey a permis de comparer les îles entre elles, lorsqu'il existe une différence significative $(\mathrm{p} \leq 0,05)$.

\section{Résultats}

\section{Richesse floristique des îles}

L'inventaire de la flore a permis de recenser au total 723 espèces végétales sur les îles. Ces espèces se répartissent en 389 genres et 99 familles. Les 17 familles les plus représentatives en nombre d'espèces sont indiquées sur la figure 2. Les familles des Fabaceae et des Rubiaceae, avec respectivement 98 et 67 espèces, sont les mieux représentées en nombre d'individus. Les autres 81 familles sont faiblement représentées, avec moins de 10 espèces chacune.

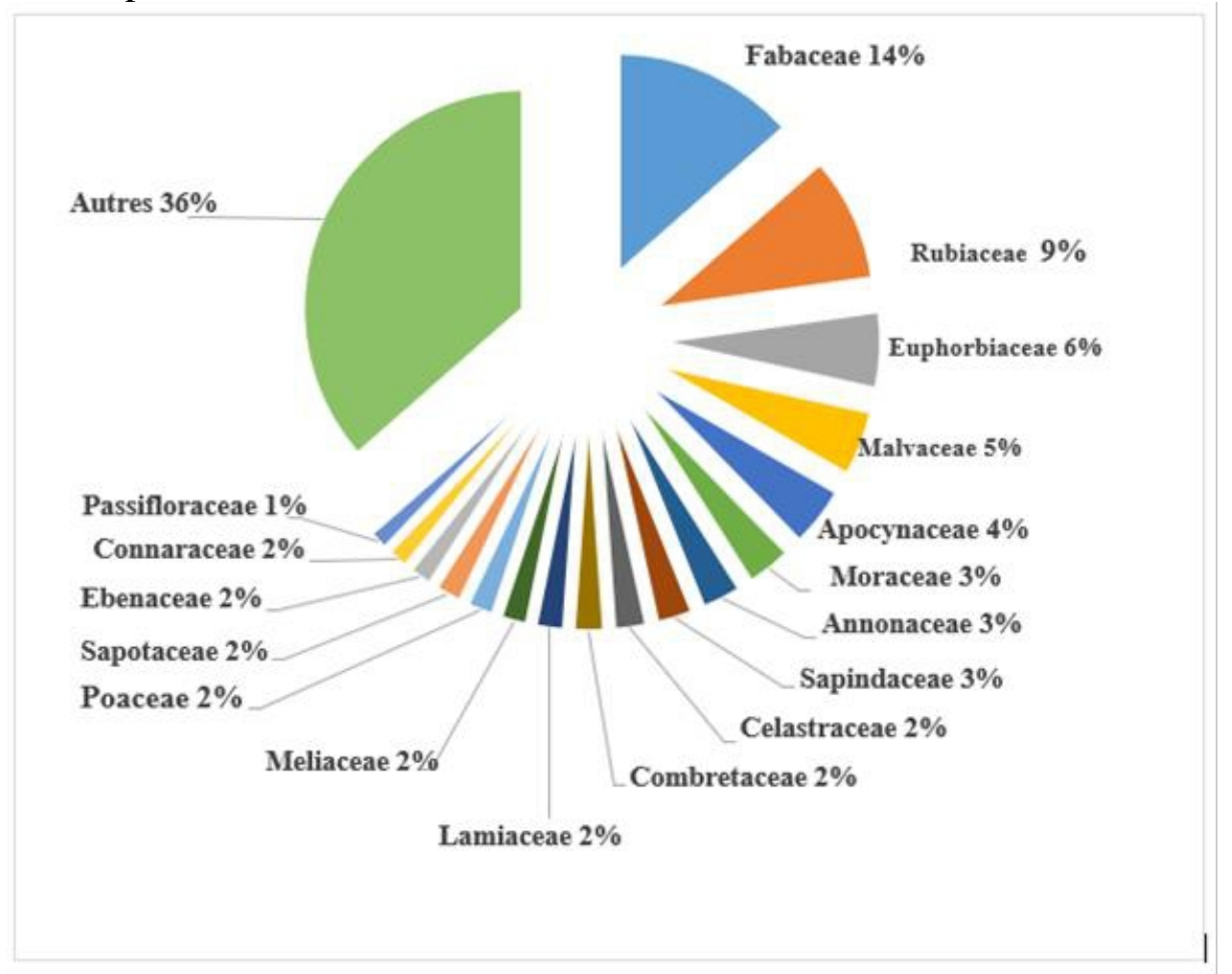

Figure 2. Spectre des familles de la flore des îles du barrage de Buyo

En considérant les relevés de surface, la moyenne du nombre d'espèces varie de façon significative $(50 \pm 6,29$ à $101 \pm 10,74)$ entre les îles. La comparaison de la richesse floristique permet de noter que l'île $\mathrm{D}$ est plus riche 
en espèces, et les îles Apollinaire et Laminebougou sont moins riches en espèces (Tableau 1).

Tableau 1. Richesse spécifique (moyenne et écart type) des différentes îles

\begin{tabular}{lcccc}
\hline \multicolumn{1}{c}{ Sites } & Superficie (Ha) & $\begin{array}{c}\text { Distances îles-forêt } \\
\text { mère }(\mathbf{m})\end{array}$ & \multicolumn{2}{c}{$\begin{array}{c}\text { Richesse spécifique } \\
\text { Total }\end{array}$} \\
& & & & $\begin{array}{c}\text { Test de Tuckey } \\
\text { p-value } \mathbf{( 0 , 0 3 7 9 7} \\
\mathbf{0 , 0 5}\end{array}$ \\
\hline Île D & 5 & 162,71 & 241 & $101 \pm 10,74^{\mathrm{a}}$ \\
\hline Île B & 10 & 244,09 & 236 & $95 \pm 13,99^{\mathrm{ab}}$ \\
\hline Île Banari & 18 & 238,10 & 218 & $93 \pm 7,45^{\mathrm{ab}}$ \\
\hline Île C & 8 & 139,35 & 255 & $89 \pm 16^{\mathrm{ab}}$ \\
\hline Île Daffibougou & 33 & 252,44 & 171 & $74 \pm 3,47^{\mathrm{ab}}$ \\
\hline Île FinDigue & 2 & 264,74 & 166 & $72 \pm 14,44^{\mathrm{ab}}$ \\
\hline Île Nacollo & 18 & 337,72 & 163 & $71 \pm 2,6^{\mathrm{ab}}$ \\
\hline Île A & 3 & 346,11 & 145 & $64 \pm 3,59^{\mathrm{ab}}$ \\
\hline Île Béatrice & 52 & 60,55 & 148 & $59 \pm 5,59^{\mathrm{ab}}$ \\
\hline Île Apollinaire & 2 & 653,14 & 124 & $51 \pm 11,14^{\mathrm{b}}$ \\
\hline Île Laminebougou & 8 & 604,98 & 126 & $50 \pm 6,29^{\mathrm{b}}$ \\
\hline
\end{tabular}

Légende: Dans les colonnes, les moyennes portant les mêmes lettres sont statistiquement égales ou semblables $(\alpha=0,05)$

\section{Composition floristique des îles}

Types biologiques, types chorologiques, types morphologiques et importance relative des espèces

Dans l'ensemble des îles, les types biologiques des espèces sont dominés par les microphanérophytes avec 196 espèces (soit $39 \%$ des espèces), que succèdent les mésophanérophytes avec 106 espèces (soit $21 \%$ ) et les nanophanérophytes, avec 89 espèces (soit $18 \%$ ) et les autres dénommés (mégaphanérophytes, thérophytes, hémicryptophytes, chaméphytes, Géophytes rhizomateuses, épiphytes, microphanérophytes (épiphytes), hydrophytes et semi-épiphytes avec 113 espèces au total (soit $22 \%$ ). Les formes lianescentes sont classées par ordre décroissant. Elles sont dominées par les lianes microphanérophytes, avec 138 espèces (soit $66 \%$ des espèces) ; les lianes mésophanérophytes avec 48 espèces (soit $23 \%$ ), les lianes mégaphanérophytes et les autres avec 22 espèces au total (soit $11 \%$ ) et 9 inconnus.

La répartition des espèces dans les régions phytogéographiques (Figure 3) montre une dominance des espèces des régions Guinéo-Congolaises (GC), avec 452 espèces (soit $63 \%$ des espèces), suivi des Guinéo-Congolaise et de Soudano-Zambèzienne (GC-SZ), avec 119 espèces (soit $17 \%$ des espèces). Parmi les espèces relevées, 115 sont reconnues comme des endémiques Ouest-africaines (GCW), dont 23 sassandriennes et 7 espèces endémiques ivoiriennes (GCi). On note également des taxons introduits ou 
cultivés (i), avec 19 espèces, (soit $3 \%$ des espèces), des taxons de la zone Soudano-Zambézienne (SZ), avec 7 espèces (soit 1\%) et 11 inconnues.

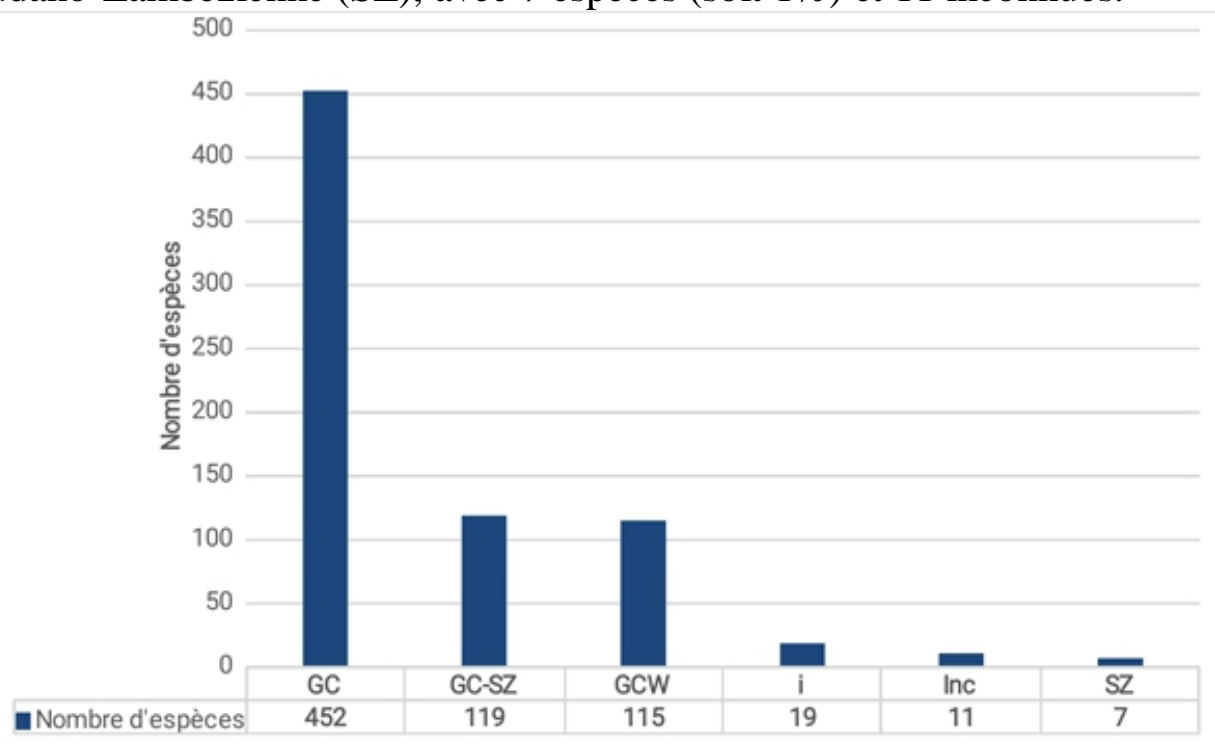

Figure 3. Répartition des espèces inventoriées sur l'ensemble des îles, par affinité chorologique.

Légende : GC = Guinéo-Congolaises ; GC-SZ = Guinéo-Congolaise et SoudanoZambèzienne ; $(\mathrm{GCW}=$ endémiques Ouest-africaines $; \mathrm{GCi}=$ endémiques ivoiriennes ; $\mathrm{i}=$ taxons introduits ou cultivés $; \mathrm{SZ}=$ Soudano-Zambézienne $; \mathrm{Inc}=$ taxons inconnus.

En considérant les arbres, arbustes et lianes de diamètres $\geq 5 \mathrm{~cm}$, nous avons inventorié au total 195 espèces végétales. Le calcul a révélé quatre espèces dont les indices d'importance sont supérieurs à 10. Nous avons, par ordre décroissant : Alchornea cordifolia, Ceiba pentandra, Theobroma cacao et Lannea welwitschii. Ces espèces constituent l'essentiel des individus de gros diamètre présents sur les îles. Les grands arbres, tels que Ceiba pentandra, Bombax buonopozense, Bombax brevicuspe, Lannea welwitschii, bien que peu représentés dans les formations forestières, doivent leur rang parmi les espèces à IVI élevé de par leur gros diamètre. Les lianes microphanérophytes, tels que Alchornea cordifolia, forment l'essentiel du sous-bois (Tableau 2).

Tableau 2. Valeurs de prépondérance des espèces des îles du barrage de Buyo

\begin{tabular}{lcccc}
\hline Espèces végétales & $\begin{array}{c}\text { Dominance } \\
\text { relative } \\
\text { (DOMesp) }\end{array}$ & $\begin{array}{c}\text { Fréquence } \\
\text { relative } \\
\text { (FREQesp) \% }\end{array}$ & $\begin{array}{c}\text { Densité } \\
\text { relative } \\
\text { (DENSesp) \% }\end{array}$ & IVI \\
\hline Alchornea cordifolia & 22,87 & 4,79 & 16,69 & 44,34 \\
\hline Ceiba pentandra & 26,56 & 4,01 & 5,16 & 35,73 \\
\hline Theobroma cacao & 13,03 & 4,27 & 12,39 & 29,69 \\
\hline Lannea welwitschii & 6,22 & 3,75 & 3,59 & 13,56 \\
\hline Ficus sur & 3,24 & 2,20 & 4,50 & 9,94 \\
\hline
\end{tabular}




\begin{tabular}{lcccc}
\hline Mangifera indica & 4,11 & 1,29 & 1,16 & 6,57 \\
\hline Maesopsis eminii & 1,57 & 2,20 & 2,23 & 5,99 \\
\hline Trema orientalis & 0,97 & 1,94 & 1,97 & 4,89 \\
\hline Myrianthus arboreus & 1,47 & 1,16 & 2,07 & 4,71 \\
\hline Morinda lucida & 0,52 & 2,46 & 1,72 & 4,70 \\
\hline Albizia zygia & 0,75 & 1,94 & 1,67 & 4,36 \\
\hline Psidium guajava & 0,44 & 1,55 & 2,23 & 4,22 \\
\hline Antiaris toxicaria & 0,35 & 2,20 & 1,62 & 4,17 \\
\hline Zanthoxylum gilletii & 0,78 & 1,68 & 1,21 & 3,68 \\
\hline Musanga cecropioides & 1,18 & 1,03 & 1,42 & 3,63 \\
\hline Ficus exasperata & 0,92 & 0,65 & 1,77 & 3,34 \\
\hline Cleistopholis patens & 0,43 & 1,29 & 1,26 & 2,99 \\
\hline Adenia cissampeloides & 0,10 & 1,81 & 0,61 & 2,51 \\
\hline Autres & 14,53 & 59,77 & 36,72 & 111,02 \\
\hline Total & $\mathbf{1 0 0}$ & $\mathbf{1 0 0}$ & $\mathbf{1 0 0}$ & $\mathbf{3 0 0}$ \\
\hline
\end{tabular}

\section{Diversité floristique des îles du barrage de Buyo}

L'indice de Shannon a montré une diversité nettement plus élevée pour l'île $\mathrm{D}$ par rapport à l'île Laminebougou. La variation de cet indice de diversité est en rapport avec le nombre d'espèces végétales. Le tableau 3 indique également que l'île $\mathrm{C}$, qui est la moins diversifiée par rapport à $\mathrm{D}$, enregistre la plus grande valeur d'indice d'équitabilité, c'est-à-dire une répartition plus homogène de sa flore. On note ainsi, que les espèces rencontrées dans l'île $\mathrm{C}$ sont plus équitablement réparties. L'île Laminebougou possède la plus petite valeur d'indice d'Équitabilité et représente l'île où les espèces sont le moins équitablement réparties, c'est-à-dire une répartition moins homogène de sa flore, due à la forte présence de Theobroma cocao et d'Alchornea cordifolia.

Tableau 3. Diversité floristique des îles du barrage de Buyo

\begin{tabular}{lcc}
\hline \multirow{2}{*}{ Sites } & \multicolumn{2}{c}{ Diversité } \\
\cline { 2 - 3 } & Shannon & Équitabilité \\
\hline Île D & $3,84 \pm 0,02$ & $0,84 \pm 0,02$ \\
\hline Île B & $3,77 \pm 0,09$ & $0,84 \pm 0,01$ \\
\hline Ille Banari & $3,60 \pm 0,14$ & $0,80 \pm 0,02$ \\
\hline Île C & $3,75 \pm 0,14$ & $0,85 \pm 0,01$ \\
\hline Île Daffibougou & $3,51 \pm 14,63$ & $0,82 \pm 0,01$ \\
\hline Île Fin Digue & $3,45 \pm 0,18$ & $0,82 \pm 0,02$ \\
\hline Île Nacollo & $3,42 \pm 0,09$ & $0,81 \pm 0,02$ \\
\hline Île A & $3.38 \pm 0,18$ & $0,81 \pm 0,03$ \\
\hline Île Béatrice & $3,21 \pm 0,24$ & $0,79 \pm 0,05$ \\
\hline Île Apollinaire & $3,04 \pm 0,28$ & $0,79 \pm 0,02$ \\
\hline Île Laminebougou & $2,99 \pm 0,21$ & $0,77 \pm 0,03$ \\
\hline
\end{tabular}




\section{Discussion \\ Richesse et composition floristique}

Cette étude botanique a permis d'identifier 723 espèces végétales reparties entre 389 genres et 99 familles. Ce nombre important d'espèces recensées au cours de la présente étude pourrait s'expliquer non seulement par le statut de protection récent dont bénéficient les îles depuis le retrait des paysans, mais aussi par la proximité de certaines de ces îles avec le Parc national de Taï. Plusieurs travaux ont révélé une grande richesse floristique du Parc national de Taï : 534 espèces (Nusbaumer, 2005), 908 espèces (Adou et al., 2005), 1300 espèces (Chatelain et al., 2001). Cette richesse pourrait aussi s'expliquer par les perturbations subies par ces îlots forestiers qui auraient favorisé la recrudescence d'espèces pionnières et secondaires (Alexandre, 1982 ; Swaine \& Hall, 1983 ; Zuidema et al., 1996 ; Laurance et al., 1998).

Les Fabaceae, les Rubiaceae, les Euphorbiaceae, les Malvaceae et les Apocynaceae sont les familles les plus représentées sur les îles du barrage de Buyo. Selon Aubreville (1959), les Rubiaceae sont l'une des familles les mieux représentées des forêts tropicales. Cela parait normal par le fait que les îles sont situées dans une zone forestière. Des études similaires ont montré une richesse spécifique des Rubiaceae, Fabaceae et Euphorbiaceae dans la forêt du Parc national de Taï (Scouppe, 2011). Cette prépondérance des Rubiaceae pourrait trouver son explication dans la position de notre zone d'étude. En effet, le Parc national de Taï se situe, avec les îles du barrage de Buyo, en zone de forêt (Région guinéo-congolaise), qui est le domaine de prédilection des Rubiaceae (Ake-Assi, 2002).

L'abondance des espèces lianescentes sur les îles s'expliquerait par le fait que toutes les îles ont été dégradées par les activités agricoles avant leur réaménagement, ce qui a favorisé l'émergence des espèces lianescentes. Les perturbations sont, selon Laurance (1991) et Kouamé (1998), propices à la prolifération des espèces lianescentes, qui sont pour la plupart des espèces secondaires. Dans la forêt classée du Haut Sassandra, Kouamé et al. (1998) ont également signalé une forte présence des espèces lianescentes sur les anciennes pistes d'exploitation. Les lianes sont signalées comme étant la forme biologique prépondérante dans les forêts tropicales en reconstitution (Devineau et al., 1984; White, 1986; Bakayoko, 1999). Leurs rôles dans la dynamique évolutive des forêts sont signalés par Schnitzer et Bongers (2002), et dans la dynamique évolutive des limites forêt-savane de Lamto (Soro et al., 2018).

Au niveau chorologique, on note la présence de $12 \%$ d'espèces ouestafricaines et $3 \%$ de sassandriennes. Les travaux d'Adou et al. (2005) ont montré que la flore de la région de Taï est riche en espèces sassandriennes. L'endémisme est communément considéré comme un critère important pour évaluer la priorité et l'état de conservation d'un espace donné. La présence en 
grand nombre de ces taxons est généralement signe d'une grande biodiversité (Adou et al., 2005). Les espèces endémiques sont hautement vulnérables aux perturbations humaines et aux autres formes de changements environnementaux, ce qui expliquerait leur faible présence sur les îles ayant connu une forte anthropisation.

\section{Diversité floristique}

La richesse et la diversité spécifique moyenne ont montré que l'île D est de loin la plus riche spécifiquement, et nettement plus diversifiée $\left(H^{\prime}=3,84 \pm 0,02\right)$. Cela pourrait s'expliquer par sa proximité avec la forêt mère, facilitant la migration d'animaux et d'oiseaux disséminateurs, en plus des nombreuses graines transportées par le vent et les courants d'eau. Selon Dorst (1947), les oiseaux granivores et frugivores contribuent à la propagation des végétaux dont ils se nourrissent des fruits et des graines. La faible activité humaine sur cette île pourrait également expliquer la forte diversité de l'île par rapport aux autres. Une étude de Sharma et Kumar (2004) réalisée en Inde, a montré que la diversité des espèces est plus élevée dans des sites moyennement perturbés.

L'île Béatrice, bien que plus proche de la forêt mère et abritant un grand nombre de bovidés, présente un faible nombre d'espèces végétales. Cette faible diversité floristique pourrait s'expliquer par la fréquentation élevée des bovidés de cette île. Plusieurs chercheurs ont qualifié l'herbivore comme étant un facteur d'altération de la végétation (Sankaran et al., 2004 ; Gignoux et al., 2009), surtout quand elle est causée par les grands mammifères (Le Roux et al., 2006). Quant aux îles Apollinaire et Laminebougou, leur faible richesse floristique constatée pourrait s'expliquer par la grande distance entre ces îles et la forêt mère. Une autre raison de leur pauvreté floristique est leur niveau de dégradation. Ces deux îles paraissent plus dégradées que les autres. Selon Bouko et al. (2007), la diversité spécifique varie en fonction du degré de dégradation. Elle est faible lorsque le milieu est fortement perturbé, et est élevée lorsque la perturbation est moindre.

\section{Conclusion}

La flore des îles du barrage de Buyo comprend 723 espèces végétales reparties entre 389 genres et 99 familles. Les Fabaceae, les Rubiaceae les Euphorbiaceae, les Malvaceae et les Apocynaceae sont les familles qui dominent les îles. Les microphanérophytes dominent en ce qui concerne les types biologiques, et les lianes microphanérophytes représentent le type morphologique le plus prépondérant sur les îles. L'île $\mathrm{D}$, qui a bénéficié d'une activité agricole de moindre intensité et est proche de la forêt mère, avec une faible présence d'animaux, est la plus riche et nettement plus diversifiée. L'île Béatrice, qui est plus proche de la forêt mère, ayant une forte présence de 
mammifères (en occurrence, les buffles), connaît une faible richesse et diversité floristique. Les îles Laminebougou et Apollinaire, très éloignées de la forêt mère et ayant subi une activité agricole intense, sont les plus pauvres en espèces végétales, et nettement moins diversifiées. Les espèces Alchornea cordifolia, Ceiba pentandra, Theobroma cacao et Lannea welwitschii sont celles qui ont un diamètre supérieur ou égal à 10 centimètres. Les îles du barrage de Buyo se classent parmi les zones les plus diversifiés des forêts denses sempervirentes en général, et des milieux aquatiques en particulier. Les relevés réalisés ont montré des variations floristiques entre les îles.

\section{Remerciements}

Nous remercions le Colonel Diarrassouba, directeur du Parc national de Taï, pour avoir permis la réalisation de cette étude. Nos remerciements vont également à l'encontre du guide et conducteur du Hors-Bord, pour nous avoir accompagné et aidé dans la réalisation de nos relevés. Nous remercions aussi chefs les des campements de pêcheurs de PK 5, PK 15, PK 28 et DERA pour leur assistance sur le terrain.

\section{Conflit d'intérêts}

Les auteurs de ce manuscrit déclarent qu'il n'y a aucun conflit d'intérêt entre eux.

\section{Contributions des Auteurs}

Dans la présente étude, SY a assuré l'élaboration du protocole de recherche, la collecte et le traitement des données, et la rédaction du manuscrit. MK a assuré une partie du traitement des données. OND et BA ont amélioré la rédaction du manuscrit par la relecture des différentes versions, pour l'amélioration de la qualité scientifique du manuscrit.

\section{References:}

1. Adou, Y. C. Y., Bakayoko, A., Akpatou, K. B. \& N'Guessan, K. (2011). Impacts de pressions anthropiques sur la flore et la structure de la végétation dans la forêt classée de Monogaga, Côte d'Ivoire. Journal of Animal \& Plant Sciences 12: 1560 - 1572.

2. Adou, Y. C. Y., Blom, E.C., Dengueadhe, K. T. S. R., Van Rompaey, R. S. A. R., N'guessan, K. E., Wittebolle, G. \& Bongers, F. (2005). Diversité floristique et végétation dans le Parc National de Taï, Côte d'Ivoire. Tropenbos-Côte d'Ivoire. Série 5: 57p.

3. Adou, Y. C. Y., N'Da, D. H., Bakayoko, A., Kouassi, K. E., N'Guessan, K. E. \& Aké-Assi, L. (2011). Distribution, Regeneration, and Conservation Status of Nuxia congesta R. Br. ex Fresen 
(Buddlejaceae) in Côte d'Ivoire. American Journal of Scientific and Industrial Research 2: 774-785.

4. Aké-Assi, L. (1984). Flore de la Côte d'Ivoire : Etude descriptive et biogéographique, avec quelques notes ethnobotaniques. Thèse de Doctorat d'Etat, de l'Université d'Abidjan, Côte d'Ivoire, $1206 \mathrm{p}$.

5. Aké-Assi, L. (2001). Flore de la Côte d'Ivoire : catalogue systématique, biogéographie et écologie. I. Boissiera 57 tome 1, 396 p.

6. Aké-Assi, L. (2002). Flore de la Côte d'Ivoire : catalogue systématique, biogéographie et écologie. II. Boissiera 58 tome 2, 401 p.

7. Alexandre, D.Y. (1982). Aspect de la régénération naturelle en forêt dense de Côte d'Ivoire. Candollea, 37: 579 - 588.

8. APG, IV. (2016). An update of the angioperm phylogeny groupe classification for the orders and families of floweringing plants. Botanical Journal of the Linnean Society 181: 1-20, https://doi.org/10.1111/boj.12385.

9. Aubréville, A. (1955). La typologie topographique forestière. Bois et Forêts des Tropiques 41: 3-7.

10. Aubreville, A. (1959). Flore forestière de la Cote d'Ivoire. Centre Technique Forêts Tropicales, Nogents / Mane, tome 1, 310 p., tome 2, 296 p., tome 3, $186 \mathrm{p}$.

11. Avenard, J.M., Eldin, M., Girad, G., Sircoulon, J., Touchebeuf, P., Guillaumet, J-L., Adjanohoum, E. \& Perraud, A. (1971). Le milieu naturel de la Cote d'Ivoire. Mémoire ORSTOM n50, Paris Franc, 392 p.

12. Bakayoko, A. (1999). Comparaison de la composition floristique et de la structure forestière des parcelles de la forêt classée de Bossématié, dans l'Est de la Côte d'Ivoire. DEA Ecologie Tropicale, Université de Cocody-Abidjan, Côte d'Ivoire. 72 p.

13. Bakayoko, A., Martin, P., Gautier, L., Chatelain, C., Traore, D. \& Spichiger, R. (2004). Etude comparative des massifs forestiers entourant la zone de Taï à Zagné (Sud-ouest de la Côte d'Ivoire). Candollea 59: 191 - 229.

14. Bakayoko, A., Chatelain, C., Martin, P., Traoré, D. \& Gautier, L. (2011). Floristic Study of Some Fragments Forests in the South Western of Côte d'Ivoire. European Journal of Scientific Research 63: $468-481$.

15. Bolgarsky, M. (1950). Etude géologique et description pétrographique du Sud-ouest de la Côte d'Ivoire. Thèse, B.R.G.M.-France, Bulletin Directeur Mines Géologies. A.O.F n 9, 205 p. 
16. Bouko, S. B, Sinsin, B. \& Soulé, G. B. (2007). Effets de la dynamique d'occupation du sol sur la structure et la diversité floristique des forêts claires et savanes au Bénin. Tropicultura 25: 221-227.

17. Chatelain, C., Gautier, L. \& Spichiger, R. (1996). A recent history of forest fragmentation in south Western Ivory Coast. Biodiversity and Conservation 5: 37-53.

18. Chatelain, C., Kadjo, B., Koné, I. \& Refisch, J. (2001). Relations Faune-Flore dans le Parc National de Taï: une étude bibliographique. Tropenbos Côte d'Ivoire 3: $166 \mathrm{p}$.

19. Collinet, J., Monteny, B. \& Pouyaud, B. (1984). Le milieu physique. In : Guillaumet J-L, Couturier G. et Dosso H. Recherche et aménagement en milieu forestier tropical humide : le projet Taï de Côte d'Ivoire. Paris : ORSTOM 15: 35- 68.

20. Corthay, R. (1996). Analyse floristique de la forêt sempervirente de Yapo (Côte d'Ivoire).Travail de Diplôme, Université de Genève, Suisse. $152 \mathrm{p}$.

21. Cottam, G. \& Curtis, J-T. (1956). The use of distance measures in phytosociological sampling. Ecology 37: 451-460.

22. Curtis, J-T. \& Mc Intoch, R. P. (1951). An upland forest continuum into prairie-forest border region wiscrusion. Ecology 32 : 476-496

23. Devineau, J-L. (1984). Structure et dynamique de quelques forêts tropophiles de l'Ouest africain (Côte d'Ivoire). In: Travaux des Chercheurs de Lamto 5: 294 p.

24. Devineau, J-L., Lecordier, C. \& Vuattoux, R. (1984). Evolution de la végétation spécifique du peuplement ligneux dans une succession préforestière de colonisation d'une savane protégée de feux (Lamto, Côte d'Ivoire). Candollea 39: 103-134.

25. Dorst, J. (1947). Le rôle disséminateur des oiseaux dans la vie des plantes. Terre et Vie 94: 106-119.

26. Eldin, M. (1971). Le climat. In : Avenard J. M., Eldin M., Gerard G., Sircoulon J., Touchebeuf P., Guillaumet J-L, Adjanohoun E. et Perraud A. (eds). Le milieu naturel de Côte d'Ivoire. Paris. 392 p.

27. FAO (2003). State of the world's forest. FAO, Rome, Italy. $151 \mathrm{p}$.

28. Gignoux, J., Lahoreau, G., Julliard, R. \& Barot, S. (2009). Establishment and early persistence of tree seedlings in an annually burned savanna. Journal of Ecology 97: 484-495.

29. Guelly, K.A. (1994). Les savanes de la zone forestière subhumide du Togo. Thèse de Doctorat, Université Pierre et Marie CURIE, Paris VI, France. 163 p.

30. Guillaumet, J. L. \& Adjanohoun, E. (1971). La végétation. In: Avenard J. M., Eldin M., Girard G., Sircoulon J., Toucheboeuf P., Guillaumet 
J-L., Adjanohoun E. et Perraud A. (eds.), Le milieu naturel de Côte d'Ivoire. Mémoire O.R.S.T.O.M. 50: 161-263.

31. Guillaumet, J. L (1967). Recherche sur la végétation et la flore de la région du Bas-Cavally (Côte-d'Ivoire). Mém. O.R.S.T.O.M. 20 p.

32. Hawksworth, D. L. \& Kalin-Arroyo, M. T. (1995). Magnitude and distribution of biodiversity. In Heywood V. H. et Waston R. T. (eds) Global biodiversity assessment: UNEP. Cambridge University Press, UK: 108-191.

33. Koffi, N. (2000). Présentation du Parc national de Taï (PNT). In: Etat des recherches en cours dans le Parc national de Taï (PNT). Sempervira 9: 5-8.

34. Kouamé, N. F. G. (1998). Influence de 1'exploitation forestière sur la végétation et la flore de la Forêt Classée du Haut Sassandra (Centre Ouest de la Côte d'Ivoire). Thèse de 3 ème cycle, Université de Cocody, Abidjan. 227 p.

35. Kouamé, D. (2008). Preliminary floristic Inventory and Diversity in Azagny National Park (Côte d'Ivoire). European journal of Scientific Research 23: 537 -547.

36. Kouamé, N'F., Tra, B. F. H., Ettien, D. T. \& Traoré, D. (1998). Végétation et flore de la forêt classée du haut Sassandra, en Côte d'Ivoire. Revue du CAMES 1: 28-35.

37. Latour, C. (1994). Etude de la biodiversité dans la parcelle 5 de l'arboretum de Jenaro Herrera (Pérou). Trav. Diplôme, Lab. Syst. Florist., Genève, 96 p.

38. Laurance, W. F., Ferreira, L.V., Rankin-De, M. J. M., Laurence, S. G., Hutchings, R. W. \& Lovejopy, T. E. (1998). Effects of forest Fragmentation on recruitment Patterns in Amazonian Tree Communities. Conservation Biology 12 : 460-464.

39. Laurance, W. F. (1991). Edge effects in tropical forest fragments : application of model for the disign of nature reserves. Biological conservation 57: 205-219.

40. Martin, C. (1991). The rainforests of West Africa: ecology, threats, conservation. Birkhauser Verlag, Bâle, Suisse. 235 p.

41. Masens, D. M. Y. (1997). Etude phytosociologique de la région de Kikwit (Bandundu, RDC), thèse de Doctorat, Fascicules Sciences, ULB Belgique. 398 p.

42. Missa, K., Ouattara, D. N., Koné, M. \& Bakayoko, A. (2015). Etude floristique et diversité de la forêt des Marais Tanoé-Ehy (Sud-Est de la Côte d'Ivoire). Journal of Animal \& Plant Sciences 25: 3917-3938.

43. Mori, S.A., Boom, B.M., De Carvalino, A.M. \& Dos Santos, T. S. (1983). Southern Bahia moist forest. Botany. Revew 49: 155-232. 
44. N'Guessan, A. J. (2001). L'impact de la création du lac de Buyo sur l'organisation de «l'espace Buyo» dans le Sud-ouest de la Côte d'Ivoire. Revue CAMES - Série B 3 : 86-96.

45. New, M., Hulme, M. \& Jones, P. (2000). Representing twentiethcentury space-time climate variability. Part II : Development of a 1901-96 mean monthly terrestrial climatology. Journal of Climate 12: 829-856.

46. N'sielolo, K. R., Lejoly, J., Habari, M. J. P. \& Aloni, K. J. (2015). Effets de lisière et de litière dans des savanes mises en défense contre les feux à Ibi village / République Démocratique du Congo. Revue Scientifique et Technique Forêt et Environnement du Bassin du Congo 5: 54-61.

47. Nusbaumer, L., Gautier, L., Chatelain, C. \& Spichiger, R. (2005). Structure et composition floristique de la forêt classée de la Scio (Côte d'Ivoire). Etude descriptive et comparative. Candollea 60: 393-443.

48. Raunkiaer, C. (1934). The life form of plants and statistical plant geography. Clarendon press, Oxford. $632 \mathrm{p}$.

49. Le Roux, X., Abbadie, L., Fritz, H. \& Leriche, H. (2006). Modification of the Savanna Fuctioning by Herbivores. In: Abbadie L, Gignoux J., Le Roux X., Lepage M. (eds) Lamto. Ecological Studies 179; 184-198.

50. Schnitzer, S. A. \& Bongers, F. (2002). The ecology of lianas and their role in forests. Trends Ecologie. \& Evolution 17: 223-230.

51. Sharma, C. M. \& Kumar, M. R. G. S. (2004). A study on community structure and diversity of a sub-tropical forest of Garhwal Himalayas. Indian Forester 130: 207- 214.

52. Scouppe, M. (2011). Composition floristique et diversité de la végétation de la zone Est du Parc National de Taï (Côte d'Ivoire). Master Université de Genève. 194 p.

53. Shannon, C.E. \& Weaver, W. (1949). The mathematic theory of communication. University Illinois Press, Urbana. 117 p.

54. Sankaran, M., Ratman, J. \& Hanan, N. (2004). Tree-grass coexistence in savannas revisited insights from an examination of assumptions and mechanisms invoked in existing models. Ecology Letters 7: 480-490.

55. Soro, Y., N'Dri, A. B., Bakayoko, A. \& Gignoux, J. (2018). Analyse de la végétation dans un écotone Forêt-Savane d'Afrique de l'Ouest dans un contexte de boisement des savanes. REB. PASRES 3: 54-72. http//www.csrs.ch/pasres: ISSN: 2520-3037.

56. Swaine, M. D. \& Hall, J. B. (1983). Early Succession on Cleared Forest Land in Ghana. Journal of Ecology 71: 601-627.

57. Van Rompaey, R. S. A. R. (1993). Forest gradients in West Africa. A spatial gradient Analysis. Agriculture University Wageningen PaysBas. 142 p. 
58. Vroh, B. T. A, Tiébré, M. S., Ouattara, D. \& N'Guessan, K. E. (2014). La réserve forestière Dékpa d'Agbaou, un exemple de conservation de la diversité végétale sur les sites miniers de la Côte d'Ivoire. International Journal of Innovation and Applied Studies 6: 162-171. http://www.ijias.issr-journals.org/.

59. White, F. (1986). La végétation de l'Afrique. Adaptation française par P. Bamps. ORTSTOM et Unesco, Paris, France, collections Recherches sur les ressources naturelles XX. 384 p.

60. Wilks, C. M. \& Issembé, Y. A. (2000). Guide pratique d'identification : les arbres de la Guinée équatoriale. Région continentale. Projet Curef, Bata. 546 p.

61. Zuidema, P. A., Sayer, J. A. \& Dijkman, W. (1996). Forest fragmentation and biodiversity: The case for intermediate-sized conservation areas. Environnement conservation 23: 287-290. 